\title{
BASINS OF ATTRACTION OF AN ANTI-COMPETITIVE DISCRETE RATIONAL SYSTEM
}

\author{
M. NURKANOVIĆ AND Z. NURKANOVIĆ
}

Dedicated to Professor Mustafa Kulenović on the occasion of his 60th birthday

ABstract. We investigate the global asymptotic behavior of solutions of the following anti-competitive system of difference equations

$$
x_{n+1}=\frac{\gamma_{1} y_{n}}{A_{1}+x_{n}}, \quad y_{n+1}=\frac{\beta_{2} x_{n}+\gamma_{2} y_{n}}{y_{n}}, \quad n=0,1, \ldots,
$$

where the parameters $\gamma_{1}, \gamma_{2}, \beta_{2}, A_{1}$ are positive numbers and the initial conditions $x_{0} \geq 0, y_{0}>0$. We find the basins of attraction of all attractors of the system, which are the equilibrium point and period-two solutions.

\section{INTRODUCTION AND MAIN RESULT}

Consider the following system of difference equations

$$
\left.\begin{array}{rl}
x_{n+1} & =\frac{\gamma_{1} y_{n}}{A_{1}+x_{n}}, \\
y_{n+1} & =\frac{\beta_{2} x_{n}+\gamma_{2} y_{n}}{y_{n}},
\end{array}\right\} \quad n=0,1, \ldots
$$

where the parameters $A_{1}, \beta_{2}, \gamma_{1}$ and $\gamma_{2}$ are positive numbers and the initial conditions $x_{0} \geq 0, y_{0}>0$.

System (1) is the first order system of difference equations of the form

$$
\left\{\begin{array}{l}
x_{n+1}=f\left(x_{n}, y_{n}\right) \\
y_{n+1}=g\left(x_{n}, y_{n}\right)
\end{array}, \quad n=0,1, \ldots,\left(x_{0}, y_{0}\right) \in \mathcal{R},\right.
$$

$\mathcal{R} \subset \mathbb{R}^{2},(f, g): \mathcal{R} \rightarrow \mathcal{R}, f, g$ are continuous functions. System (2) is competitive if $f(x, y)$ is non-decreasing in $x$ and non-increasing in $y$, and $g(x, y)$ is non-increasing in $x$ and non-decreasing in $y$. If the functions $f$ and $g$ have

2000 Mathematics Subject Classification. 37E30, 37G99, 39A10, 39A11.

Key words and phrases. Difference equations, anti-competitive, map, stability, stable set. 
monotonic character opposite of the monotonic character in competitive system, then System (2) will be called anti-competitive. It is clear that System (1) is anti-competitive.

In the classification of all linear fractional systems in [2], System (1) was mentioned as system $(16,26)$. By the change variables $v_{n}=y_{n}-\gamma_{2}$ we transform System (1) into system which is classified as $(31,16)$ and is dual of system $(16,31)$. Thus dynamics of System (1) is identical to dynamics of system $(31,16)$. Also, System (1) has a similar dynamics as system $(16,16)$, see [19].

Competitive system of the form (2) were studied by many authors and there is an extensive literature, see $[1,3,6,8,11,12,17,18,21,22]$.

The study of anti-competitive system started in [9] and has advanced since then (see $[7,10,19])$. The principal tool of study of anti-competitive systems is the fact that the second iterate of the map associated with anticompetitive system is a competitive map.

The main result of this paper, i.e. the main result on the global behavior of System (1) is the following theorem.

Theorem 1.1. a) System (1) has a unique positive equilibrium point $E=$ $(\bar{x}, \bar{y})$ for all values of the parameters.

b) If $A_{1} \gamma_{2}>\beta_{2} \gamma_{1}$, then $E$ is globally asymptotically stable, i.e. the basin of attraction of this equilibrium is $\mathcal{B}(E)=\{(x, y): x \geq 0, y>0\}$.

c) If $A_{1} \gamma_{2}<\beta_{2} \gamma_{1}$, then $E$ is a saddle point and then there exists a set $\mathcal{C} \subset \mathcal{R}$ which is an invariant subset of the basin of attraction of $E$. The set $\mathcal{C}$ is a graph of a strictly increasing continuous function of the first variable on an interval (and so is a manifold) and separates $\mathcal{R}$ into two connected and invariant components, namely

$$
\begin{aligned}
& \mathcal{W}_{-}:=\left\{\mathbf{x} \in \mathcal{R} \backslash \mathcal{C}: \exists \mathbf{y} \in \mathcal{C} \text { with } \mathbf{x} \preceq_{\text {se }} \mathbf{y}\right\} \\
& \mathcal{W}_{+}:=\left\{\mathbf{x} \in \mathcal{R} \backslash \mathcal{C}: \exists \mathbf{y} \in \mathcal{C} \text { with } \mathbf{y} \preceq_{\text {se }} \mathbf{x}\right\}
\end{aligned}
$$

which satisfy:

i) If $\left(x_{0}, y_{0}\right) \in \mathcal{W}_{+}$, then

$$
\lim _{n \rightarrow \infty}\left(x_{2 n}, y_{2 n}\right)=\left(\infty, \gamma_{2}\right) \text { and } \lim _{n \rightarrow \infty}\left(x_{2 n+1}, y_{2 n+1}\right)=(0, \infty) \text {. }
$$

ii) If $\left(x_{0}, y_{0}\right) \in \mathcal{W}_{-}$, then

$$
\lim _{n \rightarrow \infty}\left(x_{2 n}, y_{2 n}\right)=(0, \infty) \text { and } \lim _{n \rightarrow \infty}\left(x_{2 n+1}, y_{2 n+1}\right)=\left(\infty, \gamma_{2}\right) \text {. }
$$

d) Assume that $A_{1} \gamma_{2}=\beta_{2} \gamma_{1}$. Then:

i) System (1) has an infinite number of minimal period-two solutions belong to a curve

$$
x y^{2} \gamma_{1}+y\left(A_{1}+x\right)\left(x A_{1}-\gamma_{1} \gamma_{2}\right)-x \gamma_{2} A_{1}\left(A_{1}+x\right)=0 .
$$


ii) The equilibrium point $E$ and all minimal period-two solutions are stable but not asymptotically stable.

iii) There exists a family of strictly increasing curves $\mathcal{C}, \mathcal{C}_{x}$ and $\mathcal{C}^{x}$ for $x>0$, that emanate from $E, A_{x}$ and $B_{x}$, respectively, given by (10) and (11), such that the curves are pairwise disjoint, the union of all the curves equals $\mathbb{R}_{+}^{2}$, and solutions with initial point in $\mathcal{C}$ converging to $E$, solutions with initial point in $\mathcal{C}_{x}$ have even-indexed terms converging to $A_{x}$ and odd-terms converging to $B_{x}$, and, solution with initial point in $\mathcal{C}^{x}$ have even-indexed terms converging to $B_{x}$ and odd-terms converging to $A_{x}$.

\section{Preliminaries}

We now give some basic notions about competitive systems and maps in the plane of the form of (2) where $f$ and $g$ are continuous functions and $f(x, y)$ is non-decreasing in $x$ and non-increasing in $y$ and $g(x, y)$ is nonincreasing in $x$ and non-decreasing in $y$ in some domain $A$ with non-empty interior.

Consider a map $T=(f, g)$ on a set $\mathcal{R} \subset \mathbb{R}^{2}$, and let $E \in \mathcal{R}$. The point $E \in \mathcal{R}$ is called a fixed point if $T(E)=E$. An isolated fixed point is a fixed point that has a neighborhood with no other fixed points in it. A fixed point $E \in \mathcal{R}$ is an attractor if there exists a neighborhood $\mathcal{U}$ of $E$ such that $T^{n}(\mathbf{x}) \rightarrow E$ as $n \rightarrow \infty$ for $\mathbf{x} \in \mathcal{U}$; the basin of attraction is the set of all $\mathbf{x} \in \mathcal{R}$ such that $T^{n}(\mathbf{x}) \rightarrow E$ as $n \rightarrow \infty$. A fixed point $E$ is a global attractor on a set $\mathcal{K}$ if $E$ is an attractor and $\mathcal{K}$ is a subset of the basin of attraction of $E$. If $T$ is differentiable at a fixed point $E$, and if the Jacobian $J_{T}(E)$ has one eigenvalue with modulus less than one and a second eigenvalue with modulus greater than one, $E$ is said to be a saddle. See [20] for additional definitions.

Next, we give some basic facts about the monotone maps in the plane, see $[12,14,15,21]$. Now, we write System (1) in the form:

$$
\left(\begin{array}{l}
x_{n+1} \\
y_{n+1}
\end{array}\right)=T\left(\begin{array}{l}
x_{n} \\
y_{n}
\end{array}\right),
$$

where the map $T$ is given as

$$
T(x, y)=\left(\begin{array}{c}
f(x, y) \\
g(x, y)
\end{array}\right)=\left(\begin{array}{c}
\frac{\gamma_{1} y}{A_{1}+x} \\
\frac{\beta_{2} x+\gamma_{2} y}{y}
\end{array}\right) .
$$

Now, we define a partial order $\preceq$ on $\mathbb{R}^{2}$ so that the positive cone in this partial order is the fourth quadrant. Specifically, for $\mathbf{u}=\left(u_{1}, u_{2}\right), \mathbf{v}=$ $\left(v_{1}, v_{2}\right) \in \mathbb{R}^{2}$ we say that $\mathbf{u} \preceq \mathbf{v}$ if $u_{1} \leq v_{1}$ and $v_{2} \leq u_{2}$. Two points $\mathbf{u}, \mathbf{v} \in \mathbb{R}_{+}^{2}$ are said to be related if $\mathbf{u} \preceq \mathbf{v}$ or $\mathbf{v} \preceq \mathbf{u}$. 
Also, a strict inequality between points may be defined as $\mathbf{u} \prec \mathbf{v}$ if $\mathbf{u} \preceq \mathbf{v}$ and $\mathbf{u} \neq \mathbf{v}$. A stronger inequality may be defined as $\mathbf{u} \prec \prec \mathbf{v}$ if $u_{1}<v_{1}$ and $v_{2}<u_{2}$. A map $f: \operatorname{Int} \mathbb{R}_{+}^{2} \rightarrow \operatorname{Int} \mathbb{R}_{+}^{2}$ is strongly monotone if $\mathbf{u} \prec \mathbf{v}$ implies that $f(\mathbf{u}) \prec \prec f(\mathbf{v})$ for all $\mathbf{u}, \mathbf{v} \in \operatorname{Int} \mathbb{R}_{+}^{2}$. Clearly, being related is an invariant under iteration of a strongly monotone map. Differentiable strongly monotone maps have Jacobian with constant sign configuration

$$
\left[\begin{array}{cc}
+ & - \\
- & +
\end{array}\right]
$$

The mean value theorem and the convexity of $\mathbb{R}_{+}^{2}$ may be used to show that $T$ is monotone, as in [4].

For $\mathbf{x}=\left(x_{1}, x_{2}\right) \in \mathbb{R}^{2}$, define $Q_{i}(\mathbf{x})$ for $i=1, \ldots, 4$ to be the usual four quadrants based at $\mathbf{x}$ and numbered in a counterclockwise direction, for example, $Q_{1}(\mathbf{x})=\left\{\mathbf{y}=\left(y_{1}, y_{2}\right) \in \mathbb{R}^{2}: x_{1} \leq y_{1}, x_{2} \leq y_{2}\right\}$. We now state three results for competitive maps in the plane.

The following definition is from [21].

Definition 2.1. Let $\mathcal{S}$ be a nonempty subset of $\mathbb{R}^{2}$. A competitive map $T$ : $\mathcal{S} \rightarrow \mathcal{S}$ is said to satisfy condition $(O+)$ if for every $x$, y in $\mathcal{S}, T(x) \preceq_{n e} T(y)$ implies $x \preceq_{n e} y$, and $T$ is said to satisfy condition $(O-)$ if for every $x, y$ in $\mathcal{S}, T(x) \preceq_{n e} T(y)$ implies $y \preceq_{n e} x$.

The following theorem was proved by de Mottoni-Schiaffino for the Poincaré map of a periodic competitive Lotka-Volterra system of differential equations. Smith generalized the proof to competitive and cooperative maps [21].

Theorem 2.1. Let $\mathcal{S}$ be a nonempty subset of $\mathbb{R}^{2}$. If $T$ is a competitive map for which $(O+)$ holds then for all $x \in \mathcal{S},\left\{T^{n}(x)\right\}$ is eventually componentwise monotone. If the orbit of $x$ has compact closure, then it converges to a fixed point of $T$. If instead $(O-)$ holds, then for all $x \in \mathcal{S},\left\{T^{2 n}\right\}$ is eventually componentwise monotone. If the orbit of $x$ has compact closure in $\mathcal{S}$, then its omega limit set is either a period-two orbit or a fixed point.

The following result is from [21], with the domain of the map specialized to be the cartesian product of intervals of real numbers. It gives a sufficient condition for conditions $(O+)$ and $(O-)$.

Theorem 2.2. Let $\mathcal{R} \subset \mathbb{R}^{2}$ be the cartesian product of two intervals in $\mathbb{R}$. Let $T: \mathcal{R} \rightarrow \mathcal{R}$ be a $C^{1}$ competitive map. If $T$ is injective and $\operatorname{det} J_{T}(x)>0$ for all $x \in \mathcal{R}$, then $T$ satisfies $(O+)$. If $T$ is injective and $\operatorname{det} J_{T}(x)<0$ for all $x \in \mathcal{R}$, then $T$ satisfies $\left(O_{-}\right)$.

The next two results are from $[14,15]$. 
Theorem 2.3. Let $T$ be a competitive map on a rectangular region $\mathcal{R} \subset \mathbb{R}^{2}$. Let $\overline{\mathrm{x}} \in \mathcal{R}$ be a fixed point of $T$ such that $\Delta:=\mathcal{R} \cap \operatorname{int}\left(\mathcal{Q}_{1}(\overline{\mathrm{x}}) \cup \mathcal{Q}_{3}(\overline{\mathrm{x}})\right)$ is nonempty (i.e., $\overline{\mathrm{x}}$ is not the $N W$ or $S E$ vertex of $\mathcal{R}$ ), and $T$ is strongly competitive on $\Delta$. Suppose that the following statements are true:

a. The map $T$ has a $C^{1}$ extension to a neighborhood of $\overline{\mathrm{x}}$.

b. The Jacobian matrix of $T$ at $\bar{x}$ has real eigenvalues $\lambda, \mu$ such that $0<|\lambda|<\mu$, where $|\lambda|<1$, and the eigenspace $E^{\lambda}$ associated with $\lambda$ is not a coordinate axis.

Then, there exists a curve $\mathcal{C} \subset \mathcal{R}$ through $\overline{\mathrm{x}}$ that is invariant and a subset of the basin of attraction of $\overline{\mathrm{x}}$, such that $\mathcal{C}$ is tangential to the eigenspace $E^{\lambda}$ at $\overline{\mathrm{x}}$, and $\mathcal{C}$ is the graph of a strictly increasing continuous function of the first coordinate on an interval. Any endpoints of $\mathcal{C}$ in the interior of $\mathcal{R}$ are either fixed points or minimal period-two points. In the latter case, the set of endpoints of $\mathcal{C}$ is a minimal period-two orbit of $T$.

Theorem 2.4. Let $\mathcal{I}_{1}, \mathcal{I}_{2}$ be intervals in $\mathbb{R}$ with endpoints $a_{1}, a_{2}$ and $b_{1}, b_{2}$ with endpoints respectively, with $a_{1}<a_{2}$ and $b_{1}<b_{2}$, where $-\infty \leq a_{1}<$ $a_{2} \leq \infty$ and $-\infty \leq b_{1}<b_{2} \leq \infty$. Let $T$ be a competitive map on an rectangle $\mathcal{R}=\mathcal{I}_{1} \times \mathcal{I}_{2}$ and $\overline{\mathbf{x}} \in \operatorname{int}(\mathcal{R})$. Suppose that the following hypotheses are satisfied:

1. $T(\operatorname{int}(\mathcal{R})) \subset \operatorname{int}(\mathcal{R})$ and $T$ is strongly competitive on int $(\mathcal{R})$.

2. The point $\overline{\mathbf{x}}$ is the only fixed point of $T$ in $\left(Q_{1}(\overline{\mathbf{x}}) \cup Q_{3}(\overline{\mathbf{x}})\right) \cap$ int $(\mathcal{R})$.

3. The map $T$ is continuously differentiable in a neighborhood of $\overline{\mathbf{x}}$.

4. At least one of the following statements is true.

a. $T$ has no minimal period two orbits in $\left(Q_{1}(\overline{\mathbf{x}}) \cup Q_{3}(\overline{\mathbf{x}})\right) \cap$ int $(\mathcal{R})$.

b. $\operatorname{det} J_{T}(\overline{\mathbf{x}})>0$ and $T(\mathbf{x})=\overline{\mathbf{x}}$ only for $\mathbf{x}=\overline{\mathbf{x}}$.

5. $\overline{\mathbf{x}}$ is a saddle point.

Then, the following statements are true:

(i.) The stable set $\mathcal{W}^{s}(\overline{\mathbf{x}})$ is connected and it is the graph of a continuous increasing curve with endpoints in $\partial \mathcal{R}$. int $(\mathcal{R})$ is divided by the closure of $\mathcal{W}^{s}(\overline{\mathbf{x}})$ into two invariant connected regions $\mathcal{W}_{+}$("below the stable set"), and $\mathcal{W}_{-}$("above the stable set"), where

$$
\begin{aligned}
& \mathcal{W}_{-}:=\left\{\mathbf{x} \in \mathcal{R} \backslash \mathcal{W}^{s}(\overline{\mathbf{x}}): \exists \mathbf{x}^{\prime} \in \mathcal{W}^{s}(\overline{\mathbf{x}}) \text { with } \mathbf{x} \preceq_{s e} \mathbf{x}^{\prime}\right\}, \\
& \mathcal{W}_{+}:=\left\{\mathbf{x} \in \mathcal{R} \backslash \mathcal{W}^{s}(\overline{\mathbf{x}}): \exists \mathbf{x}^{\prime} \in \mathcal{W}^{s}(\overline{\mathbf{x}}) \text { with } \mathbf{x}^{\prime} \preceq_{s e} \mathbf{x}\right\} .
\end{aligned}
$$

(ii.) The unstable set $\mathcal{W}^{u}(\overline{\mathbf{x}})$ is connected and it is the graph of a continuous decreasing curve.

(iii.) For every $\mathbf{x} \in \mathcal{W}_{+}, T^{n}(\mathbf{x})$ eventually enters the interior of the invariant set $Q_{4}(\overline{\mathbf{x}}) \cap \mathcal{R}$, and for every $\mathbf{x} \in \mathcal{W}_{-}, T^{n}(\mathbf{x})$ eventually enters the interior of the invariant set $Q_{2}(\overline{\mathbf{x}}) \cap \mathcal{R}$. 
(iv.) Let $\mathbf{m} \in Q_{2}(\overline{\mathbf{x}})$ and $\mathbf{M} \in Q_{4}(\overline{\mathbf{x}})$ be the endpoints of $\mathcal{W}^{u}(\overline{\mathbf{x}})$, where $\mathbf{m} \preceq_{\text {se }} \overline{\mathbf{x}} \preceq_{\text {se }} \mathbf{M}$. For every $\mathbf{x} \in \mathcal{W}_{-}$and every $\mathbf{z} \in \mathcal{R}$ such that $\mathbf{m} \preceq_{\text {se }} z$, there exists $m \in \mathbb{N}$ such that $T^{m}(\mathbf{x}) \preceq_{\text {se }} z$, and for every $\mathbf{x} \in \mathcal{W}_{+}$and every $\mathbf{z} \in \mathcal{R}$ such that $\mathbf{z} \preceq_{\text {se }} \mathbf{M}$, there exists $m \in \mathbb{N}$ such that $\mathbf{M} \preceq_{\text {se }} T^{m}(\mathbf{x})$.

The following result gives a convergence result for a system in $\mathbb{R}^{2}$ when there exists an invariant rectangle, and the map of the system is an anticompetitive and satisfies certain conditions. See [10].

Theorem 2.5. Let $T$ be an anti-competitive map on a closed and bounded rectangular region $\mathcal{R} \subset \mathbb{R}^{2}$. Suppose that $T$ has a unique fixed point $\overline{\mathbf{e}}$ in $\mathcal{R}$ and that $T$ has no minimal period-two solutions. Then, $\overline{\mathbf{e}}$ is globally asymptotically stable on $\mathcal{R}$.

\section{LiNEARIZED STABILITY ANALYSIS}

Theorem 3.1. System (1) has the unique positive equilibrium point $E$ for all values of the parameters.

i) If $A_{1} \gamma_{2}-\beta_{2} \gamma_{1}>0$, then $E$ is locally asymptotically stable.

ii) If $A_{1} \gamma_{2}-\beta_{2} \gamma_{1}<0$, then $E$ is a saddle point.

iii) If $A_{1} \gamma_{2}-\beta_{2} \gamma_{1}=0$, then $E$ is non-hyperbolic.

Proof. The equilibrium point $E=(\bar{x}, \bar{y})$ of System (1) satisfies the following system of equations:

$$
\bar{x}=\frac{\gamma_{1} \bar{y}}{A_{1}+\bar{x}}, \bar{y}=\frac{\beta_{2} \bar{x}+\gamma_{2} \bar{y}}{\bar{y}},
$$

i.e.

$$
\bar{x}^{2}=\gamma_{1} \bar{y}-A_{1} \bar{x}, \quad \bar{y}^{2}=\beta_{2} \bar{x}+\gamma_{2} \bar{y} .
$$

This implies that System (1) has a unique positive equilibrium point $E=$ $(\bar{x}, \bar{y})$, which is an intersection of the following two parabolas:

$$
\bar{y}=\frac{1}{\gamma_{1}}\left(\bar{x}^{2}+A_{1} \bar{x}\right), \bar{x}=\frac{1}{\beta_{2}}\left(\bar{y}^{2}-\gamma_{2} \bar{y}\right) .
$$

The map $T$ associated to System (1) is (3) and the Jacobian matrix of $T$ at the equilibrium point $E=(\bar{x}, \bar{y})$ is

$$
J_{T}(\bar{x}, \bar{y})=\left(\begin{array}{cc}
-\frac{\gamma_{1} \bar{y}}{\left(A_{1}+\bar{x}\right)^{2}} & \frac{\gamma_{1}}{A_{1}+\bar{x}} \\
\frac{\beta_{2}}{\bar{y}} & -\frac{\beta_{2} \bar{x}}{\bar{y}^{2}}
\end{array}\right)=\left(\begin{array}{cc}
-\frac{\bar{x}^{2}}{\gamma_{1} \bar{y}} & \frac{\bar{x}}{\bar{y}} \\
\frac{\beta_{2}}{\bar{y}} & -\frac{\beta_{2} \bar{x}}{\bar{y}^{2}}
\end{array}\right) .
$$

The corresponding characteristic equation is of the form:

$$
\lambda^{2}-p \lambda+q=0
$$


where

$$
\begin{aligned}
& p=\operatorname{Tr} J_{T}(E)=-\frac{\bar{x}^{2}}{\gamma_{1} \bar{y}}-\frac{\beta_{2} \bar{x}}{\bar{y}^{2}}<0, \\
& q=\operatorname{Det}_{T}(E)=\frac{\beta_{2} \bar{x}^{3}}{\gamma_{1} \bar{y}^{3}}-\frac{\beta_{2} \bar{x}}{\bar{y}^{2}}=\frac{\beta_{2} \bar{x}}{\gamma_{1} \bar{y}^{3}}\left(\bar{x}^{2}-\gamma_{1} \bar{y}\right)=-\frac{A_{1} \beta_{2} \bar{x}^{2}}{\gamma_{1} \bar{y}^{3}}<0 .
\end{aligned}
$$

i) It is obvious that $1+q<2$. On other hand:

$$
\begin{aligned}
|p|< & 1+q \Leftrightarrow \frac{\bar{x}^{2}}{\gamma_{1} \bar{y}}+\frac{\beta_{2} \bar{x}}{\bar{y}^{2}}<1+\frac{\beta_{2} \bar{x}^{3}}{\gamma_{1} \bar{y}^{3}}-\frac{\beta_{2} \bar{x}}{\bar{y}^{2}} \\
& \stackrel{(4)}{\Leftrightarrow} \frac{\gamma_{1} \bar{y}-A_{1} \bar{x}}{\gamma_{1} \bar{y}}+\frac{\beta_{2} \bar{x}}{\bar{y}^{2}}<1+\frac{\beta_{2} \bar{x}^{3}}{\gamma_{1} \bar{y}^{3}}-\frac{\beta_{2} \bar{x}}{\bar{y}^{2}} \Leftrightarrow-A_{1} \bar{y}^{2}+2 \beta_{2} \gamma_{1} \bar{y}<\beta_{2} \bar{x}^{2} \\
& \stackrel{(4)}{\Leftrightarrow}-A_{1}\left(\beta_{2} \bar{x}+\gamma_{2} \bar{y}\right)+2 \beta_{2} \gamma_{1} \bar{y}<\beta_{2}\left(\gamma_{1} \bar{y}-A_{1} \bar{x}\right) \Leftrightarrow A_{1} \gamma_{2}>\beta_{2} \gamma_{1},
\end{aligned}
$$

which implies that $E$ is locally asymptotically stable if $A_{1} \gamma_{2}>\beta_{2} \gamma_{1}$.

ii) Now, we check conditions for $E$ to be a saddle point.

(1) $p^{2}-4 q>0$ because $q<0$.

(2) $|p|>|1+q| \Leftrightarrow p^{2}>(1+q)^{2} \Leftrightarrow(p-1-q)(p+1+q)>0$.

$$
1^{\circ} p-1-q=-\frac{\bar{x}^{2}}{\gamma_{1} \bar{y}}-\frac{\beta_{2} \bar{x}}{\bar{y}^{2}}-1-\frac{\beta_{2} \bar{x}^{3}}{\gamma_{1} \bar{y}^{3}}+\frac{\beta_{2} \bar{x}}{\bar{y}^{2}}=-\frac{\bar{x}^{2}}{\gamma_{1} \bar{y}}-1-\frac{\beta_{2} \bar{x}^{3}}{\gamma_{1} \bar{y}^{3}}<0,
$$

$2^{\circ}$ We see that i) implies: $p+1+q>0 \Leftrightarrow A_{1} \gamma_{2}-\beta_{2} \gamma_{1}>0$, from which

$$
p+1+q<0 \Leftrightarrow A_{1} \gamma_{2}<\beta_{2} \gamma_{1} .
$$

iii) It is obvious that

$$
|p|=|1+q| \Leftrightarrow A_{1} \gamma_{2}=\beta_{2} \gamma_{1}
$$

i.e. now $E$ is a non-hyperbolic equilibrium point.

\section{Period-two solutions}

In this section, we present the results for the existence of period-two solutions of System (1).

Lemma 4.1. If $A_{1} \gamma_{2} \neq \beta_{2} \gamma_{1}$, then System (1) has no minimal period-two solutions.

Proof. System (1) can be reduced to the following second-order difference equation:

$$
x_{n+2}=\gamma_{1} \frac{\gamma_{2} A_{1} x_{n+1}+\gamma_{2} x_{n} x_{n+1}+\beta_{2} \gamma_{1} x_{n}}{\left(A_{1}+x_{n+1}\right) x_{n+1}\left(A_{1}+x_{n}\right)},
$$

or to the following second-order difference equation:

$$
y_{n+2}=\frac{\gamma_{2} y_{n} y_{n+1}^{2}-\gamma_{2}^{2} y_{n} y_{n+1}+\beta_{2}^{2} \gamma_{1} y_{n}+\beta_{2} \gamma_{2} A_{1} y_{n+1}}{\left(y_{n} y_{n+1}+\beta_{2} A_{1}-\gamma_{2} y_{n}\right) y_{n+1}}
$$


Now, we prove that both of the difference equations (7) and (8) have no minimal period-two solutions. Assume that this is not true for equation (7), that is that

$$
\varphi, \psi, \varphi, \psi, \ldots,(\varphi \neq \psi)
$$

is a minimal period-two solution of (7). Then, we have:

$$
\begin{aligned}
& \varphi=\gamma_{1} \frac{\gamma_{2} A_{1} \psi+\gamma_{2} \varphi \psi+\beta_{2} \gamma_{1} \varphi}{\left(A_{1}+\psi\right) \psi\left(A_{1}+\varphi\right)}, \\
& \psi=\gamma_{1} \frac{\gamma_{2} A_{1} \varphi+\gamma_{2} \psi \varphi+\beta_{2} \gamma_{1} \psi}{\left(A_{1}+\varphi\right) \varphi\left(A_{1}+\psi\right)}
\end{aligned}
$$

from which:

$$
\begin{aligned}
& \varphi \psi\left(A_{1}+\psi\right)\left(A_{1}+\varphi\right)=\gamma_{1}\left(\gamma_{2} A_{1} \psi+\gamma_{2} \varphi \psi+\beta_{2} \gamma_{1} \varphi\right), \\
& \varphi \psi\left(A_{1}+\psi\right)\left(A_{1}+\varphi\right)=\gamma_{1}\left(\gamma_{2} A_{1} \varphi+\gamma_{2} \psi \varphi+\beta_{2} \gamma_{1} \psi\right) .
\end{aligned}
$$

By subtraction, we obtain

$$
(\psi-\varphi)\left(A_{1} \gamma_{2}-\beta_{2} \gamma_{1}\right)=0,
$$

and this implies that $\psi=\varphi$, which is a contradiction.

Now, assume that

$$
\chi, \phi, \chi, \phi, \ldots,(\chi \neq \phi)
$$

is a minimal period-two solution of equation (8). Then, we have:

$$
\begin{aligned}
& \chi=\frac{\gamma_{2} \chi \phi^{2}-\gamma_{2}^{2} \chi \phi+\beta_{2}^{2} \gamma_{1} \chi+\beta_{2} \gamma_{2} A_{1} \phi}{\left(\chi \phi+\beta_{2} A_{1}-\gamma_{2} \chi\right) \phi}, \\
& \phi=\frac{\gamma_{2} \phi \chi^{2}-\gamma_{2}^{2} \phi \chi+\beta_{2}^{2} \gamma_{1} \phi+\beta_{2} \gamma_{2} A_{1} \chi}{\left(\phi \chi+\beta_{2} A_{1}-\gamma_{2} \phi\right) \chi} .
\end{aligned}
$$

This implies:

$$
\begin{aligned}
& \left(\chi \phi+\beta_{2} A_{1}-\gamma_{2} \chi\right) \phi \chi=\gamma_{2} \chi \phi^{2}-\gamma_{2}^{2} \chi \phi+\beta_{2}^{2} \gamma_{1} \chi+\beta_{2} \gamma_{2} A_{1} \phi, \\
& \left(\phi \chi+\beta_{2} A_{1}-\gamma_{2} \phi\right) \chi \phi=\gamma_{2} \phi \chi^{2}-\gamma_{2}^{2} \phi \chi+\beta_{2}^{2} \gamma_{1} \phi+\beta_{2} \gamma_{2} A_{1} \chi .
\end{aligned}
$$

By subtracting, we obtain:

$$
\left(A_{1} \gamma_{2}-\beta_{2} \gamma_{1}\right)(\phi-\chi)=0,
$$

from which $\phi=\chi$. It is a contradiction.

Lemma 4.2. If $A_{1} \gamma_{2}=\beta_{2} \gamma_{1}$, then System (1) has continuum of minimal period-two solutions $A_{x}$ and $B_{x}$, for $x>0$, of the form:

$$
\begin{gathered}
A_{x}=\left(x, \frac{-\left(A_{1}+x\right)\left(x A_{1}-\gamma_{1} \gamma_{2}\right)+\sqrt{\left(A_{1}+x\right)^{2}\left(x A_{1}-\gamma_{1} \gamma_{2}\right)^{2}+4 x^{2} \gamma_{1} \gamma_{2} A_{1}\left(A_{1}+x\right)}}{2 x \gamma_{1}}\right), \\
B_{x}=\left(x_{B}, y_{B}\right),
\end{gathered}
$$


where

$$
\begin{gathered}
x_{B}=\frac{-\left(A_{1}+x\right)\left(x A_{1}-\gamma_{1} \gamma_{2}\right)+\sqrt{\left(A_{1}+x\right)^{2}\left(x A_{1}-\gamma_{1} \gamma_{2}\right)^{2}+4 x^{2} \gamma_{1} \gamma_{2} A_{1}\left(A_{1}+x\right)}}{2 x\left(A_{1}+x\right)}, \\
y_{B}=\frac{\left(x+A_{1}\right)\left(x A_{1}+\gamma_{1} \gamma_{2}\right)+\sqrt{\left(A_{1}+x\right)^{2}\left(x A_{1}-\gamma_{1} \gamma_{2}\right)^{2}+4 x^{2} \gamma_{1} \gamma_{2} A_{1}\left(A_{1}+x\right)}}{2 \gamma_{1}\left(x+A_{1}\right)} .
\end{gathered}
$$

Proof. Minimal period-two solution of System (1) is a fixed point of the map $T^{2}$, which is equivalent to the following system

$$
\left\{\begin{array}{l}
u(x, y)=x \\
v(x, y)=x
\end{array}\right.
$$

i.e.

$$
\begin{gathered}
\left\{\begin{array}{l}
\frac{\left(x \beta_{2}+y \gamma_{2}\right) \gamma_{1}\left(x+A_{1}\right)}{y\left(A_{1}^{2}+x A_{1}+y \gamma_{1}\right)}=x \\
\frac{y^{2} \beta_{2} \gamma_{1}+\left(y \gamma_{2}+x \beta_{2}\right) \gamma_{2}\left(x+A_{1}\right)}{\left(x \beta_{2}+y \gamma_{2}\right)\left(x+A_{1}\right)}=y
\end{array}\right. \\
\Leftrightarrow\left\{\begin{array}{l}
\left(x \beta_{2}+y \gamma_{2}\right) \gamma_{1}\left(x+A_{1}\right)=x y\left(A_{1}^{2}+x A_{1}+y \gamma_{1}\right) \\
y^{2} \beta_{2} \gamma_{1}+\left(y \gamma_{2}+x \beta_{2}\right) \gamma_{2}\left(x+A_{1}\right)=y\left(x \beta_{2}+y \gamma_{2}\right)\left(x+A_{1}\right)
\end{array}\right. \\
\Leftrightarrow\left\{\begin{array}{l}
\left(x \beta_{2}+y \gamma_{2}\right) \gamma_{1}\left(x+A_{1}\right)=x y\left(A_{1}^{2}+x A_{1}+y \gamma_{1}\right) \\
y\left(-y \gamma_{1}+x A_{1}+x^{2}\right)\left(\beta_{2} \gamma_{1}-\gamma_{2} A_{1}\right)=0 .
\end{array}\right.
\end{gathered}
$$

It is easy to see that $x>0$ and $y>0$. Since $x \neq \bar{x}, y \neq \bar{y}$, from the second equation in (14) we obtain $A_{1} \gamma_{2}=\beta_{2} \gamma_{1}$. Now, the first equation in (14) is of the form:

$$
x y^{2} \gamma_{1}+y\left(A_{1}+x\right)\left(x A_{1}-\gamma_{1} \gamma_{2}\right)-x \gamma_{2} A_{1}\left(A_{1}+x\right)=0 .
$$

So, System (1) has infinitely many minimal period-two solutions which are located along the third order curve given by (15), i.e.

$\mathcal{G}=\left\{(x, y) \in(0, \infty)^{2}: x y^{2} \gamma_{1}+y\left(A_{1}+x\right)\left(x A_{1}-\gamma_{1} \gamma_{2}\right)-x \gamma_{2} A_{1}\left(A_{1}+x\right)=0\right\}$.

It is easy to see that $A_{x}, B_{x} \in \mathcal{G}$ and that $T\left(A_{x}\right)=B_{x}, T\left(B_{x}\right)=A_{x}$ if $A_{1} \gamma_{2}=\beta_{2} \gamma_{1}$.

\section{Global Results}

In this section, we present results about basins of attraction of System (1).

Lemma 5.1. The map $T^{2}$ is injective and $\operatorname{det} J_{T^{2}(x, y)}>0$ for all $x \geq 0$ and $y>0$. 
Proof. 1) The map $T^{2}$ is of the form:

$$
\begin{aligned}
T^{2}(x, y) & =(u(x, y), v(x, y)) \\
& =\left(\frac{\left(x \beta_{2}+y \gamma_{2}\right) \gamma_{1}\left(x+A_{1}\right)}{y\left(A_{1}^{2}+x A_{1}+y \gamma_{1}\right)}, \frac{y^{2} \beta_{2} \gamma_{1}+\left(y \gamma_{2}+x \beta_{2}\right) \gamma_{2}\left(x+A_{1}\right)}{\left(x \beta_{2}+y \gamma_{2}\right)\left(x+A_{1}\right)}\right) .
\end{aligned}
$$

Now, we prove that the map $T^{2}$ is injective. Indeed, $T\left(x_{1}, y_{1}\right)=T\left(x_{2}, y_{2}\right)$ implies that

$$
A_{1}\left(y_{1}-y_{2}\right)=x_{1} y_{2}-x_{2} y_{1}=0,
$$

from which we obtain $y_{1}=y_{2}$ and $x_{1}=x_{2}$, i.e. $\left(x_{1}, y_{1}\right)=\left(x_{2}, y_{2}\right)$. This implies that $T$ is injective and that $T^{2}$ is injective, too.

2 ) For the map $T^{2}(x, y)=(u(x, y), v(x, y))$ we have:

$$
J_{T^{2}}(x, y)=\left(\begin{array}{ll}
u_{x} & u_{y} \\
v_{x} & v_{y}
\end{array}\right)
$$

where

$$
\begin{aligned}
& u_{x}=\frac{\left(\beta_{2} A_{1}^{3}+y^{2} \gamma_{1} \gamma_{2}+2 x \beta_{2} A_{1}^{2}+x^{2} \beta_{2} A_{1}+2 x y \beta_{2} \gamma_{1}+y \beta_{2} \gamma_{1} A_{1}\right) \gamma_{1}}{y\left(y \gamma_{1}+x A_{1}+A_{1}^{2}\right)^{2}}, \\
& u_{y}=-\frac{\gamma_{1}\left(x+A_{1}\right)\left(y^{2} \gamma_{1} \gamma_{2}+x \beta_{2} A_{1}^{2}+x^{2} \beta_{2} A_{1}+2 x y \beta_{2} \gamma_{1}\right)}{y^{2}\left(y \gamma_{1}+x A_{1}+A_{1}^{2}\right)^{2}}, \\
& v_{x}=-y^{2} \beta_{2} \gamma_{1} \frac{2 x \beta_{2}+y \gamma_{2}+\beta_{2} A_{1}}{\left(x \beta_{2}+y \gamma_{2}\right)^{2}\left(x+A_{1}\right)^{2}}, \quad v_{y}=\frac{y \beta_{2} \gamma_{1}\left(2 x \beta_{2}+y \gamma_{2}\right)}{\left(x+A_{1}\right)\left(x \beta_{2}+y \gamma_{2}\right)^{2}} .
\end{aligned}
$$

After some simplifications, we obtain:

$$
\operatorname{det} J_{T^{2}}=\frac{\beta_{2}^{2} \gamma_{1}^{2} A_{1}^{2}}{\left(y \gamma_{1}+x A_{1}+A_{1}^{2}\right)^{2}\left(x \beta_{2}+y \gamma_{2}\right)}>0
$$

for all $x \geq 0$ and $y>0$, and the Jacobian matrix of $T^{2}(x, y)$ is invertible.

Remark 5.1. By Lemma 5.1 and Theorems 2.1 and 2.2 we see that the map $T^{2}$ satisfies condition $(O+)$ and consequently, the sequences $\left\{x_{2 n}\right\},\left\{x_{2 n+1}\right\}$, $\left\{y_{2 n}\right\},\left\{y_{2 n+1}\right\}$ of every solution of System (1) are eventually monotone.

Lemma 5.2. The map $T^{2}$ associated to System (1) satisfied the following:

$$
T^{2}(x, y)=(\bar{x}, \bar{y}) \quad \text { only for }(x, y)=(\bar{x}, \bar{y}) .
$$

Proof. Since $T^{2}$ is injective, then

$$
T^{2}(x, y)=(\bar{x}, \bar{y})=T^{2}(\bar{x}, \bar{y}) \Rightarrow(x, y)=(\bar{x}, \bar{y}) .
$$




\section{Proof of Theorem 1.1.}

Case 1: $A_{1} \gamma_{2}>\beta_{2} \gamma_{1}$

Since $f(x, y)$ is decreasing in $x$ and increasing in $y$, while $g(x, y)$ is increasing in $x$ and decreasing in $y$, we have:

$$
\begin{aligned}
0 & \leq x_{n+1}=\frac{\gamma_{1} y_{n}}{A_{1}+x_{n}} \leq \frac{\gamma_{1} U_{2}}{A_{1}} \leq U_{1}, \\
\gamma_{2} \leq y_{n+1} & =\frac{\beta_{2} x_{n}+\gamma_{2} y_{n}}{y_{n}}=\frac{\beta_{2} x_{n}}{y_{n}}+\gamma_{2} \leq \frac{\beta_{2} U_{1}}{\gamma_{2}}+\gamma_{2} \leq U_{2},
\end{aligned}
$$

which implies:

$$
U_{1} \geq \frac{\gamma_{1} U_{2}}{A_{1}} \geq \frac{\gamma_{1}}{A_{1}}\left(\frac{\beta_{2} U_{1}}{\gamma_{2}}+\gamma_{2}\right) \Leftrightarrow U_{1} \geq \frac{\gamma_{1} \gamma_{2}^{2}}{A_{1} \gamma_{2}-\beta_{2} \gamma_{1}}>0
$$

and

$$
U_{2} \geq \frac{\beta_{2} U_{1}}{\gamma_{2}}+\gamma_{2} \geq \frac{\beta_{2} \gamma_{1} \gamma_{2}}{A_{1} \gamma_{2}-\beta_{2} \gamma_{1}}+\gamma_{2}=\frac{A_{1} \gamma_{2}^{2}}{A_{1} \gamma_{2}-\beta_{2} \gamma_{1}}>0
$$

i.e.

$$
U_{1} \geq \frac{\gamma_{1} \gamma_{2}^{2}}{A_{1} \gamma_{2}-\beta_{2} \gamma_{1}}, U_{2} \geq \frac{A_{1} \gamma_{2}^{2}}{A_{1} \gamma_{2}-\beta_{2} \gamma_{1}} .
$$

This shows that

$$
\left[0, U_{1}\right] \times\left[\gamma_{2}, U_{2}\right]
$$

is an attractive box.

Now, we show that $\mathcal{R}=\left[0, U_{1}^{*}\right] \times\left[\gamma_{2}, U_{2}^{*}\right]$, where $U_{1}^{*}=\frac{\gamma_{1} \gamma_{2}^{2}}{A_{1} \gamma_{2}-\beta_{2} \gamma_{1}}$ and $U_{2}^{*}=\frac{A_{1} \gamma_{2}^{2}}{A_{1} \gamma_{2}-\beta_{2} \gamma_{1}}$, is an invariant box, i.e. $T(\mathcal{R}) \subset \mathcal{R}$. Indeed, suppose that $(x, y) \in \mathcal{R}$. Then, we have:

$$
\begin{gathered}
0 \leq f(x, y)=\frac{\gamma_{1} y}{A_{1}+x} \leq \frac{\gamma_{1} y}{A_{1}} \leq \frac{\gamma_{1} U_{2}^{*}}{A_{1}}=U_{1}^{*}, \\
\gamma_{2} \leq g(x, y)=\frac{\beta_{2} x}{y}+\gamma_{2} \leq \frac{\beta_{2} U_{1}^{*}}{y}+\gamma_{2}=U_{2}^{*} .
\end{gathered}
$$

By Lemma 4.1 and Theorem 2.5 equilibrium $E=(\bar{x}, \bar{y})$ is globally asymptotically stable.

Case 2: $A_{1} \gamma_{2}<\beta_{2} \gamma_{1}$

It is easy to check that $E=(\bar{x}, \bar{y})$ is a saddle point for $T^{2}$ as well. System (1) can be decomposed into the system of the even-indexed and odd-indexed 
terms as follows:

$$
\left\{\begin{array}{l}
x_{2 n+1}=\frac{\gamma_{1} y_{2 n}}{A_{1}+x_{2 n}}, \\
x_{2 n}=\frac{\gamma_{1} y_{2 n-1}}{A_{1}+x_{2 n-1}}, \\
y_{2 n+1}=\frac{\beta_{2} x_{2 n}}{y_{2 n}}+\gamma_{2}, \\
y_{2 n}=\frac{\beta_{2} x_{2 n-1}}{y_{2 n-1}}+\gamma_{2} .
\end{array}\right.
$$

The existence of the set $\mathcal{C}$ with stated properties follows from Lemmas 5.1, 4.1, 4.2 and 5.2 and Theorems 2.3 and 2.4.

Case 3: $A_{1} \gamma_{2}=\beta_{2} \gamma_{1}$

Lemma 5.3. Assume that $\beta_{2} \gamma_{1}=A_{1} \gamma_{2}$. Then, the following statements are true.

i) All periodic points $A_{x}, B_{x} \in \mathcal{G}$, given by (10) and (11), are nonhyperbolic fixed points for the map $T^{2}$, and in both of them the corresponding Jacobian matrix of the the map $T^{2}$ has eigenvalues $\lambda_{1}=1$ and $\lambda_{2} \in(0,1)$.

ii) Eigenvectors corresponding to the eigenvalues $\lambda_{1}$ and $\lambda_{2}$ are not parallel to coordinate axes.

Proof. i) From (16), we obtain

$$
\begin{aligned}
& y_{\mathcal{G}-}(x)=\frac{-\left(A_{1}+x\right)\left(x A_{1}-\gamma_{1} \gamma_{2}\right)-\sqrt{\left(A_{1}+x\right)^{2}\left(x A_{1}-\gamma_{1} \gamma_{2}\right)^{2}+4 x^{2} \gamma_{1} \gamma_{2} A_{1}\left(A_{1}+x\right)}}{2 x \gamma_{1}}, x \neq 0, \\
& y_{\mathcal{G}+}(x)=\frac{-\left(A_{1}+x\right)\left(x A_{1}-\gamma_{1} \gamma_{2}\right)+\sqrt{\left(A_{1}+x\right)^{2}\left(x A_{1}-\gamma_{1} \gamma_{2}\right)^{2}+4 x^{2} \gamma_{1} \gamma_{2} A_{1}\left(A_{1}+x\right)}}{2 x \gamma_{1}}, x \neq 0,
\end{aligned}
$$

and $y_{\mathcal{G}-}(x)<0, y_{\mathcal{G}+}(x)>0$ for $x>0$.

The curve $y_{\mathcal{G}}(x)$ is decreasing in $x$, that is $y_{\mathcal{G}+}^{\prime}(x)<0$. Indeed, from (12) we have:

$$
\begin{aligned}
& u_{x}+u_{y} y^{\prime}=1, \\
& v_{x}+v_{y} y^{\prime}=y^{\prime}
\end{aligned}
$$

If $(x, y) \in \mathcal{G}$, then

where

$$
y_{\mathcal{G}+}^{\prime}(x)=\frac{1-\Gamma}{\Lambda}=\frac{\Theta}{1-\Omega},
$$

$$
\Gamma:=u_{x}(x, y), \Lambda:=u_{y}(x, y), \Theta:=v_{x}(x, y), \Omega:=v_{y}(x, y) .
$$

Since $\Theta<0$ for $x>0$, it is sufficient to prove that $\Omega<1$. Namely, if $\beta_{2} \gamma_{1}=A_{1} \gamma_{2}$, then we have:

$$
\begin{aligned}
\Omega & =\frac{y \beta_{2} \gamma_{1}\left(2 x \beta_{2}+y \gamma_{2}\right)}{\left(x+A_{1}\right)\left(x \beta_{2}+y \gamma_{2}\right)^{2}}=\frac{y A_{1} \gamma_{2}\left(2 x \beta_{2}+y \gamma_{2}\right)}{\left(x+A_{1}\right)\left(x \beta_{2}+y \gamma_{2}\right)^{2}}<1 \\
& \Leftrightarrow x\left(x^{2} \beta_{2}^{2}+y^{2} \gamma_{2}^{2}+x \beta_{2}^{2} A_{1}+2 x y \beta_{2} \gamma_{2}\right)>0,
\end{aligned}
$$


which is satisfied for all $x>0$ and $y>0$.

Since $\Gamma>0, \Lambda<0$ and $y_{\mathcal{G}+}^{\prime}(x)=\frac{1-\Gamma}{\Lambda}=\frac{\Theta}{1-\Omega}<0$, we have that $\Gamma \in(0,1)$. The characteristic polynomial of the matrix

$$
J_{T^{2}}^{\mathcal{G}}(x, y)=\left(\begin{array}{cc}
\Gamma & \Lambda \\
\Theta & \Omega
\end{array}\right)
$$

is of the form:

$$
P(\lambda)=\lambda^{2}-(\Gamma+\Omega) \lambda+(\Gamma \Omega-\Lambda \Theta)=\lambda^{2}-\left(\lambda_{1}+\lambda_{2}\right) \lambda+\lambda_{1} \lambda_{2} .
$$

On other hand, $(20)$ implies that $(1-\Gamma)(1-\Omega)=\Lambda \Theta$, i.e.

$$
P(1)=1-(\Gamma+\Omega)+(\Gamma \Omega-\Lambda \Theta)=0 .
$$

This means that $\lambda_{1}=1$. So, $\lambda_{1}+\lambda_{2}=1+\lambda_{2}=\Gamma+\Omega<2$, from which follows that $\lambda_{2}<1$. Since $\lambda_{1} \lambda_{2}=\Gamma \Omega-\Lambda \Theta>0$ and $\lambda_{1}=1$, we have that $\lambda_{2}>0$.

ii) The eigenvectors $\mathbf{v}_{1}=\left(v_{1}^{1}, v_{2}^{1}\right)$ and $\mathbf{v}_{2}=\left(v_{1}^{2}, v_{2}^{2}\right)$, corresponding to the eigenvalues $\lambda_{1}=1$ and $\lambda_{2}=\Gamma \Omega-\Lambda \Theta$, are of the following form:

$$
\mathbf{v}_{1}=\left(\begin{array}{c}
1-\Omega \\
\Theta
\end{array}\right) \text { and } \mathbf{v}_{2}=\left(\begin{array}{c}
\Gamma-1 \\
\Theta
\end{array}\right) .
$$

Since $\Gamma \in(0,1), \Lambda<0, \Theta<0$ and $\Omega \in(0,1)$, we see that the eigenvectors are not parallel to coordinate axes.

Therefore all conditions of Theorem 2.3 are satisfied for the map $T^{2}$ with $\mathcal{R}=[0, \infty) \times(0, \infty)$.

\section{REFERENCES}

[1] A. Brett, M. Garić-Demirović, M. R. S. Kulenović and M. Nurkanović, Global behavior of two competitive rational systems of difference equations in the plane, Comm. Appl. Nonlinear Anal., 16 (2009), 1-18.

[2] E. Camouzis, M. R. S. Kulenović, G. Ladas and O. Merino, Rational systems in the plane-open problems and conjectures, J. Difference Equ. Appl., 15 (2009), 303-323.

[3] D. Clark and M. R. S. Kulenović, On a coupled system of rational difference equations, Comput. Math. Appl., 43 (2002), 849-867.

[4] D. Clark, M. R. S. Kulenović, and J. F. Selgrade, Global asymptotic behavior of a two dimensional difference equation modelling competition, Nonlinear Anal., TMA, 52 (2003), 1765-1776.

[5] C. A. Clark, M. R. S. Kulenović, and J.F. Selgrade, On a system of rational difference equations, J. Difference Equ. Appl., 11 (2005), 565-580.

[6] M. Garic-Demirović, M. R. S. Kulenović and M. Nurkanović, Global behavior of four competitive rational systems of difference equations in the plane, Discrete Dyn. Nat. Soc., (2009), Art. ID 153058, 34 pages. 
[7] M. Garić-Demirović and M. Nurkanović, Dynamics of an anti-competitive two dimensional rational system of difference equations, Sarajevo J. Math., 7 (19) (2011), $39-56$.

[8] M. Hirsch and H. Smith, Monotone Dynamical Systems, Handbook of Differential Equations, Ordinary Differential Equations, (second volume), 239-357, Elsevier B. V., Amsterdam, 2005.

[9] S. Kalabušić and M. R. S. Kulenović, Dynamics of certain anti-competitive systems of rational difference equations in the plane, J. Difference Equ. Appl., 17 (2011), 1599-1615.

[10] S. Kalabušić, M. R. S. Kulenović and E. Pilav, Global dynamics of anti-competitive systems in the plane, (submitted).

[11] M. R. S. Kulenović and O. Merino, Discrete Dynamical Systems and Difference Equations with Mathematica, Chapman and Hall/CRC, Boca Raton, London, 2002.

[12] M. R. S. Kulenović and O. Merino, Competitive-exclusion versus competitivecoexistence for systems in the plane, Discrete Cont. Dyn. Syst. Ser. B, 6 (2006), $1141-1156$.

[13] M. R. S. Kulenović and O. Merino, A global attractivity result for maps with invariant boxes, Discrete Cont. Dyn. Syst. Ser. B, 6 (2006), 97-110.

[14] M. R. S. Kulenović and O. Merino, Global bifurcation for discrete competitive systems in the plane, Discrete Cont. Dyn. Syst. Ser. B, 12 (2009), 133-149.

[15] M. R. S. Kulenović and O. Merino, Invariant manifolds for competitive discrete systems in the plane, Internat. J. Bifur. Chaos Appl. Sci. Engrg., 20 (8) (2010), 24712486 .

[16] M. R. S. Kulenović and M. Nurkanović, Asymptotic behavior of two dimensional linear fractional system of difference equations, Rad. Mat., 11 (2002), 59-78.

[17] M. R. S. Kulenović and M. Nurkanović, Asymptotic behavior of a competitive system of linear fractional difference equations, J. Inequal. Appl., 2 (2005), 127-144.

[18] M. R. S. Kulenović and M. Nurkanović, Asymptotic behavior of a system of linear fractional difference equations, Adv. Difference Equ., (2006), Art. ID 19756, 13 pp.

[19] M. R. S. Kulenović and M. Nurkanović, Basins of attraction of an anti-competitive system of difference equations, Comm. Appl. Nonlinear Anal., 19 (2) (2012), 41-53.

[20] C. Robinson, Stability, Symbolic Dynamics, and Chaos, CRC Press, Boca Raton, 1995.

[21] H. L. Smith, Planar competitive and cooperative difference equations, J. Difference Equ. Appl., 3 (1998), 335-357.

[22] H. L. Smith, The discrete dynamics of monotonically decomposable maps, J. Math. Biology, 53 (2006), 747-758.

(Received: July 9, 2012)

Department of Mathematics

University of Tuzla

Univerzitetska 4, 75000 Tuzla

Bosnia and Herzegovina

E-mail: mehmed.nurkanovic@untz.ba

zehra.nurkanovic@untz.ba 